\title{
Facing the Challenges of Pharmaceutical Research Using Whispering Gallery Modes-Based Biosensors
}

\author{
Jimena Álvarez Freile ${ }^{1,2}$, Ghizlane Choukrani ${ }^{1,2}$, Edwin Bremer ${ }^{2}$ and Lars Dähne ${ }^{1 *}$ \\ ${ }^{1}$ Surflay Nanotec GmbH, Germany \\ ${ }^{2}$ Department of Haematology, University Medical Center Groningen, The Netherlands
}

*Corresponding author: Lars Dähne, Surflay Nanotec GmbH, Max-Planck-Str.3, 12489 Berlin, Tel: 0049-30-63921764 Germany.

To Cite This Article: Lars Dähne, Facing the Challenges of Pharmaceutical Research Using Whispering Gallery Modes-Based Biosensors. 2020 - 8(2). AJBSR.MS.ID.001259. DOI: 10.34297/AJBSR.2020.08.001259.

Received: 阱 March 09, 2020; Published: 眥 March 19, 2020

\begin{abstract}
During the last decade, different Whispering Gallery Modes-based biosensors (WGMS) have been proposed as bioanalytical platforms. These systems allow for a label-free real-time monitoring of a wide range of molecules, including DNA, proteins and polymers. With the advancement of new technologies and the increasing complexity of novel biotherapeutics, the development of versatile tools is of great relevance to meet the needs of the pharmaceutical research. Throughout this review, the role of WGMS in the drug development process and their ability to face the current challenges and limitations in this field will be discussed.
\end{abstract}

Keywords: Whispering Gallery Modes, Biosensors, Label-free detection, Drug development

Abbrevations: WGMS: Whispering Gallery Modes-based Sensors, SPR: Surface Plasmon Resonance, KA: Association Constant, KD: Dissociation Constant

\section{Introduction}

Optical biosensors are well-known analytical tools for probing biomolecular interactions between molecules. These sensors have a wide range of applications including medical diagnostics (e.g. cancer biomarker detection) and drug development [1]. In the last years, there has been a growing interest for label-free technologies, which present intrinsic advantages over their label-based counterparts, including real-time kinetic analysis, faster development and no bio-affinity alteration due to the incorporated label [2]. There are several label free technologies, including Whispering Gallery Modes-based biosensors (WGMS). The basic principle of WGM relies upon total internal reflection of light trapped inside a circular resonator which circulates along the surface, producing associated photons with certain wavelengths which can be detected as narrow peaks in the optical spectrum [3]. Any modification in the optical geometry or refractive index on the surrounding environment (e.g. binding of molecules to the resonator surface) induces a shift in WGM wavelength positions, which can be correlated with the total number of bound molecules. Due to the small diameters of the circular resonators between 200 and $7 \mu \mathrm{m}$, they can detect less molecules and can be used in miniaturized devices [4]. The specificity for the analyte detection relies on surface immobilized molecules (e.g. antibodies, membrane receptors) which react selectively with the analyte of interest. Several strategies have been described for surface immobilization, being either random or sitedirected (e.g. by protein A, G) [5].

\section{Use of WGMS in (pre)clinical drug development}

One critical parameter that needs to be assessed during the drug development process is the binding profile of biotherapeutics to their target molecule (e.g. association (KA) and dissociation (KD) constants). An example of WGMS for determining the KD of the biotin-streptavidin binding pair was reported by Soteropulus et al. [6]. In this study, the KD was estimated from a linearization of the dissociation phase in good agreement with previous values in the literature. However, efforts need to be made to reduce mass transport limitations problems, for example by optimizing the flow rate and ligand concentration, for an accurately determination of the KA. Additionally, for kinetic analysis each binding pair 
should be tested empirically to evaluate the most suitable sitedirected immobilization method in order to control the density of binding sites on the sensor surface, as very high one might alter the KD and KA values. On the other hand, the increasing complexity of novel biotherapeutics has raised new challenges for functional characterization, as when compared to standard antibodies, bispecific antibodies require the consideration of two individual interactions [7]. Only a couple of technologies, including suspension array technology [8] or surface plasmon resonance (SPR) have been described for bi-functional characterization in one approach. Meschendoerfer et al. have proposed a SPR-based dual binding assay in which a bispecific antibody is immobilized on the SPR chip through a capture antibody [9]. Despite their promising results, the capture antibody introduces an additional interaction that is not totally desirable. While current SPR systems are ideal for characterizing single interaction pairs, they might expertise difficulties resolving multi-variant interactions due to the limited number of available configurations and the lack of controlled immobilization of ligands on the sensor surface [10]. To the best of our knowledge, WGMS haven't been described for bi-functional characterization although their versatility for surface immobilization of molecules on the sensor surface and the possibility of using geometrical cell-like-microparticles as sensors, offering a more 3D realistic interaction setting, makes this technology an attractive alternative for functional analysis of new bi-functional therapeutic agents.

\section{Use of WGMS for real-time monitoring of therapy response}

Another important stage in the drug development process is the in vitro assessment of cell responses after drug treatment. Chen et al. described in 2016 a WGMS for the specific and labelfree detection of procaspase- 3 released from human cell lines after treatment with a toxin [11]. The same group has recently improved the system by integrating WGMS with a microfluidic platform for detecting cytochrome c, a soluble biomarker of apoptosis, with nanomolar sensitivity in cell culture media after treatment of cell lines in the same way [12]. Although several parameters such as cell culture perturbation or the limit of detection still need to be fully assessed, these initial studies suggests a potential role of WGMS for the non-invasive real time monitoring of biomarkers release from intact and nonlabelled cell cultures, which might be also implemented within complex organ-on-a-chip systems.

\section{Conclusion}

Some of the most important challenges and applications of WGMS within the biopharmaceutical research have been discussed. Although more efforts need to be done to improve WGMS competitiveness, WGMS are a worthy technology to look at for facing the needs of the biopharmaceutical and biotechnological research and overcome some of the limitations of current methods.

The work was supported by the European Commission under GA number 813871 — I-Direct.

\section{References}

1. Kim E, Baaske MD, Vollmer F (2017) Towards next-generation label-free biosensors: recent advances in whispering gallery mode sensors. Lab on a Chip vol 17(7): 1190-1205.

2. Quinn MK, Gnan N, James S, Ninarello A, Sciortino F, et al. (2015) How fluorescent labelling alters the solution behaviour of proteins. Phys Chem Chem Phys 17(46): 31177-31187.

3. Francois A, Himmelhaus M (2009) Optical sensors based on whispering gallery modes in fluorescent microbeads: Size dependence and influence of substrate. Sensors 9(9): 6836-6852.

4. Olszyna M, Debrassi A, Üzüm C, Dähne L (2018) Label-Free Bioanalysis Based on Low-Q Whispering Gallery Modes: Rapid Preparation of Microsensors by Means of Layer-by-Layer Technology. Adv Funct Mater 29(2): 1-7.

5. Makaraviciute A, Ramanaviciene A (2013) Site-directed antibody immobilization techniques for immunosensors. Biosensors and Bioelectronics 50: 460-471.

6. Soteropulos CE, Hunt HK, Armani AM (2011) Determination of binding kinetics using whispering gallery mode microcavities. Appl Phys Lett 99(10): 2012-2015.

7. Shim H (2020) Bispecific Antibodies and Antibody-Drug Conjugates for Cancer Therapy: Technological Considerations. Biomolecules 10(3).

8. Leng Y, Sun K, Chen X, Li W (2015) Suspension arrays based on nanoparticle-encoded microspheres for high-throughput multiplexed detection. Chem Soc Rev 44(15): 5552-5595.

9. Meschendoerfer W, Gassner C, Lipsmeier F, Regula JT, Moelleken J (2017) SPR-based assays enable the full functional analysis of bispecific molecules. J Pharm Biomed Anal 132: 141-147.

10. Abbas A, Linman MJ, Cheng Q (2011) New trends in instrumental design for surface plasmon resonance-based biosensors. Biosens Bioelectron 26: $1815-1824$.

11. Chen YJ, Xiang W, Klucken J, Vollmer F (2016) Tracking MicroOptical Resonances for Identifying and Sensing Novel procaspase-3 Protein Marker Released from Cell Cultures in Response to Toxins. Nanotechnology 27(16): 164001.

12. Chen YJ, Schoeler U, Huang CHB, Vollmer F (2018) Combining Whispering-Gallery Mode Optical Biosensors with Microfluidics for Real-Time Detection of Protein Secretion from Living Cells in Complex Media. Small 14(22): e1703705. 